\title{
Model of Power Soft MnZn Ferrites, Including Temperature Effects
}

\author{
L. Zegadi, Jean Jacques Rousseau, Bruno Allard, Member, IEEE, Pierre Tenant, and D. Renault
}

\begin{abstract}
This paper describes a model for simulating the behavior of soft MnZn. This model takes into account both hysteresis and dynamic phenomena. The temperature is introduced using behavioral laws. In fact, the following model requires only few parameters. It estimates iron losses and characteristics, such as $B(H), \varphi(t)$, and the induced electromotive force. The obtained results are compared with measured data for three soft MnZn ferrites currently used in power electronics. The comparison found good agreement in a wide range of operating frequencies for temperatures ranging from 40 to $140{ }^{\circ} \mathrm{C}$.
\end{abstract}

\section{INTRODUCTION}

$\mathbf{T}$ HE miniaturization of power converters requires higher operating frequencies and smaller magnetic components. The converter efficiency depends on both power semiconductor losses and losses from magnetic devices. A great consideration has been given to the modeling of the behavior of power semiconductor devices. In this respect, powerful simulators such as SPICE, SABER [1], or PACTE [2] have been developed. However, the modeling of the magnetic components has not yet received full attention. The performance of magnetic components is known to depend strongly on the choice of both the core material and the winding. Usually, magnetic materials are characterized by hysteresis and dynamic effects.

In power electronics, magnetic components operate under given conditions. First, the flux waveform is more often triangular or trapezoidal, symmetrical (push pull application) or asymmetrical (forward, flyback) with sometimes a high DC level (inductors used in forward converter). Second, the operating frequency ranges between $10 \mathrm{kHz}$ and $1 \mathrm{MHz}$, and the maximum flux density is limited by the temperature of the magnetic component.

For this purpose, polycrystalline soft MnZn ferrites materials are used. They have a high magnetic permeability and a high resistivity to eddy currents. There are many grades and types of ferrites; nearly all of them may be classified into relatively few categories according to the main applications for which they are intended. The operating frequency may be increased by a reduction of the grain size, the control of microstructure homogeneity, and by a higher isolating grain boundary [3]. The magnetic characteristics of soft ferrites depend strongly on temperature. Increased losses cause the core temperature to rise and approach the Curie temperature (in the range of $180-250{ }^{\circ} \mathrm{C}$ ) [4]. The

\footnotetext{
Manuscript received March 28, 1998; revised November 18, 1999. This work was supported by the French Ministry of Research and Space.

The authors are with CEGELY-INSA, UMR CNRS 5005, F-69621 Villeurbanne Cedex, France (e-mail: allard@cegely.insa-lyon.fr).

Publisher Item Identifier S 0018-9464(00)05851-9.
}

curve showing the magnetic losses as a function of the temperature presents a minimum at a temperature that corresponds to the maximum permeability $\left(80\right.$ to $\left.100{ }^{\circ} \mathrm{C}\right)$. The variation of the temperature modifies all of the characteristics of soft ferrites. The evolution of the main characteristics versus the temperature are similar among all of the tested soft ferrites [5]. Increasing the temperature decreases the saturation flux density, the remanence, and the coercive force. It is then extremely important for a designer to have a practical model for estimating the losses under various excitations and temperatures. Such a model should also be easy to use for industrial applications. Its main features are the simplicity and rapidity to obtain results with an adequate accuracy. Moreover, it should give waveforms and requires few parameters to be identified. The magnetic component losses have contributions from both the core and the winding. Losses caused by the winding have been considered by many authors [6]-[8]. Litz wire, foil, and printed conductors are used to reduce these losses. However, modeling the core losses in specific conditions that may be met in power electronics is not yet available. The difficulty in modeling core losses is mainly because of the difficulty in modeling hysteresis losses, which is one of the two parts of the total core losses. It should be noted that considerable efforts have been devoted to develop models of hysteresis: the most commonly quoted are the Preisach [9] and Jiles [10] models. The latter is used in two simulators: SPICE and SABER. These models are not satisfactorily suitable for soft ferrites in power electronics because they do not take into account the temperature. (The Jiles and other models are satisfactory without temperature effect consideration for other applications.) We have developed and improved the Preisach model, which is adapted to soft ferrites and enables us to introduce the temperature as a variable.

The aim of this paper is to study a model of the behavior of soft ferrites that takes into account the temperature. It describes accurately the hysteresis phenomena that predominate in low frequency (high-level flux density applications) and dynamic effects. This model suits a wide range of operating frequencies for arbitrary waveforms (symmetric or asymmetric). The model requires two parameters: a behavioral coefficient $\gamma$ and a static function $B(H)$. To take the temperature into consideration, the model is firstly set at the temperature of minimum loss. The model parameters are then computed at this particular temperature and kept independent of the temperature. Additional empirical laws are introduced to take care of the temperature dependencies of the flux density and the remanence.

The proposed model needs only few parameters that can be easily obtained through simple measurements or from the manufacturer. The simulated results are finally compared with experi- 


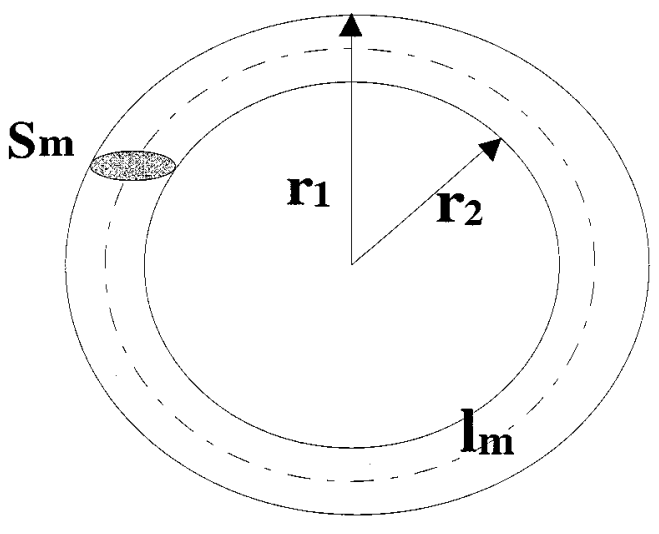

Fig. 1. Uniform toroid.

mental results on three industrial soft ferrites, and a good agreement is found in a wide range of operating frequencies and flux density levels. The temperature range considered is between 40 and $140{ }^{\circ} \mathrm{C}$ (at high temperature, the material approaches the Curie point; therefore, saturation flux density is too weak). The model may accurately predict the iron losses and the waveforms: $B(H), \varphi(t)$, as well as the induced electromotive force (e.m.f).

\section{The Behavioral Model at FiXed Temperature}

\section{A. Principle of the Model}

The magnetic behavior of a conducting circuit changes both according to the frequency and waveform of the applied magnetic field. Modeling the circuit magnetic behavior may consist in using an equivalent circuit representation. We consider a uniform toroid having a small radial thickness. For such a core, it may be assumed that the field strength is uniform and that the magnetic path length and cross-sectional area equal the mean circumference and physical cross-sectional area, respectively (Fig. 1) [11], [12].

The modeling technique is based on the fact that an insulating magnetic circuit may be satisfactorily described by a static characteristic $B(H)$. For a conductive circuit, it is assumed that local eddy currents or dynamic properties may be represented by a lumped fictitious winding of " $n$ " turns shorted by a resistor " $r$ " on an insulating magnetic circuit with the same magnetic characteristics (Fig. 2).

Considering the equivalent circuit [Fig. 2(b)], the following relation is obtained (Ampere law):

$$
\sum N I=N I_{\mathrm{appl}}+n i \text {. }
$$

$I_{\text {appl }}$ is the applied current, and $i$ is the induced current. The sum of the applied and the fictitious current verifies the quasistatic characteristic.

The induced current is given by the relation

$$
i=\frac{e}{r}=-\frac{n}{r} \frac{d \varphi}{d t} .
$$

The total magnetic field is

$$
H_{r}=H_{\mathrm{appl}}+\frac{n i}{l}
$$

or, introducing (2)

$$
H_{r}=H_{\mathrm{appl}}-\frac{n^{2}}{l_{m .} .} \frac{d \varphi}{d t} .
$$

The variation of the instantaneous flux is given by the relation

$$
\frac{d \varphi}{d t}=\frac{r \cdot l_{m}}{n^{2}}\left[H_{\mathrm{appl}}-H_{r}\right] .
$$

Let $\gamma=n^{2} / r ;$ then

$$
\frac{d \varphi}{d t}=\frac{l_{m}}{\gamma}\left[H_{\mathrm{appl}}-H_{r}\right] .
$$

The variation of the flux density is given by the following relation:

$$
\frac{d B}{d t}=\frac{l_{m}}{S_{m}} \frac{1}{\gamma}\left[H_{\mathrm{appl}}-F^{-1}(B)\right]
$$

where $l_{m}$ and $S_{m}$ are the mean length and the mean cross-sectional area of the magnetic circuit, respectively.

$\gamma=n^{2} / r$ is a behavioral coefficient and is obtained by identification procedure, and $F^{-1}(B)=H$ is the static function.

\section{B. Evaluating the Value of the Behavioral Coefficient $\gamma$}

The evaluation procedure is carried out by using first magnetization curves. For the same value of the applied field, two measurements are achieved: a static first magnetization curve and a dynamic first magnetization curve at a given frequency (the dynamic curve would serve as a reference curve). The simulation is achieved using the model (7), where the static function $F^{-1}(B)$ is introduced as a static first magnetization curve and the applied field $H_{\text {appl }}$ as the applied field that has been used in the measurement of the dynamic first magnetization curve. The obtained simulated curve is then compared with the measured dynamic curve. The selected coefficient $\gamma$ enables us to reproduce the dynamic first magnetization curve in the operating flux density levels (50 to $300 \mathrm{mT}$ ). An optimization criterion based on the measured and simulated losses is considered

$$
\varepsilon=\frac{\int_{0}^{H_{1}}\left(B_{\text {meas }}(H)-B_{\text {sim }}(H)\right) d H}{\int_{0}^{H_{1}} B_{\text {meas }}(H) d H} .
$$

The behavioral coefficient $\gamma$ is chosen so that $\varepsilon(\%)$ is less then $10 \%$. Fig. 3 shows the results obtained at $80^{\circ} \mathrm{C}$ with a behavioral coefficient of 0.045 for the $\mathrm{B} 2$ material.

First, we have investigated the dependence of the behavioral coefficient $\gamma$ versus frequency: as a result, a constant $\gamma$ enables the reproduction of the dynamic characteristics in a wide range of frequencies and waveforms. We have also investigated the dependence of the behavioral coefficient $\gamma$ versus the cross-sectional area. For a given material, tests have been carried out for toroids with approximately the same effective magnetic length and height but different cross-sectional area ranging for 27 to $110 \mathrm{~mm}^{2}$. As a result, a behavioral coefficient $\gamma_{1}$, where $\gamma_{1}=$ $\left(S_{e} / l_{e}\right) \gamma$, identified for one core, enables the accurate calculation of the dynamic characteristics for toroids with different cross-sectional areas, but the same material. The value $\gamma_{1}$ is specific for each given material. 
Conductive mate rial
(Distributed current)

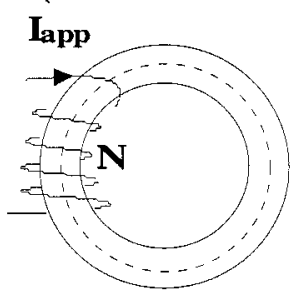

(a)

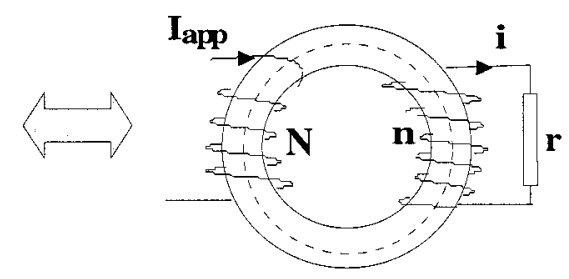

(b)

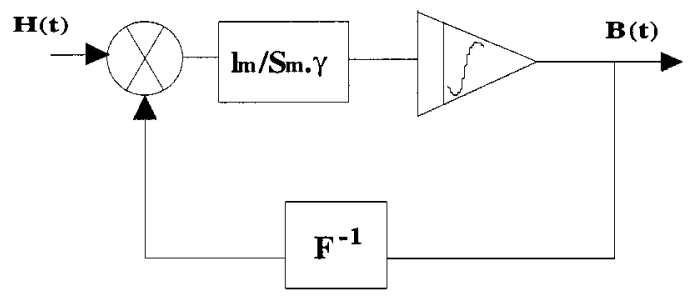

(c)

Fig. 2. Principle of the model and the corresponding simulation diagram: (a) circuit considered for simulation, (b) principle of the model, and (c) simulation diagram.

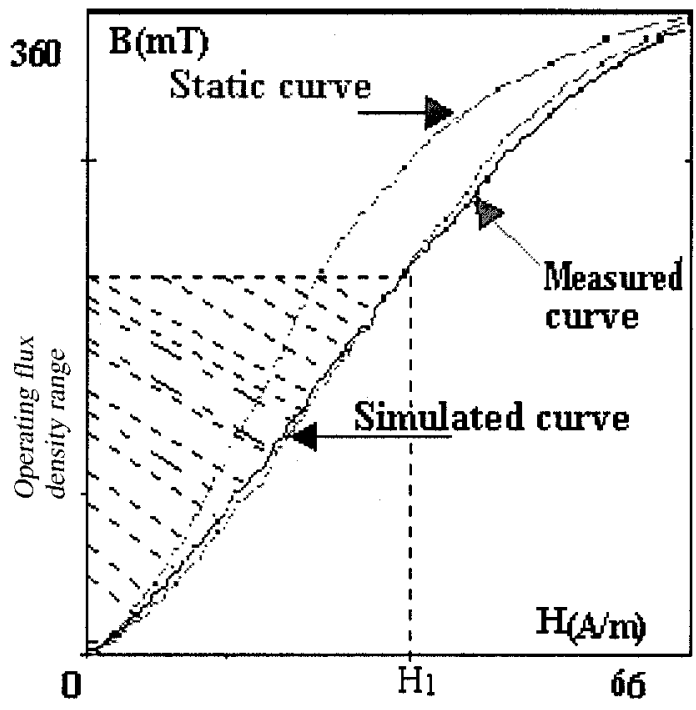

Fig. 3. Identification procedure of the behavioral coefficient.

\section{The Static Function}

$F^{-1}(B)=H$ or $B=F(H)$ represents the static function. In power electronics, the waveforms can be symmetrical or asymmetrical. To achieve these conditions, an improved Preisach-Néel model is used. The Preisach-Néel model is favored for its simplicity in describing these waveforms. The discrete form proposed by Biorci and Pescetti [13] has been used. The model requires a first static magnetization curve in combination with the descending saturation hysteresis loop. For soft ferrite materials, the Preisach-Néel model is accurate in describing the high-level hysteresis loops, but its accuracy decreases significantly at low flux density levels. The model predicts accurately the maximum flux density, but a large difference is found between the measured and simulated remanences and coercive forces. Fig. 4(a) shows a comparison between a simulated and a measured descending hysteresis loop.

Some studies have been carried out on the Preisach model [14]-[16]. It has been established that the model computes the irreversible part of magnetization, but unsatisfactorily describes the reversible part, which is important in the case of low flux density. This is because the slopes after the turning points as de-

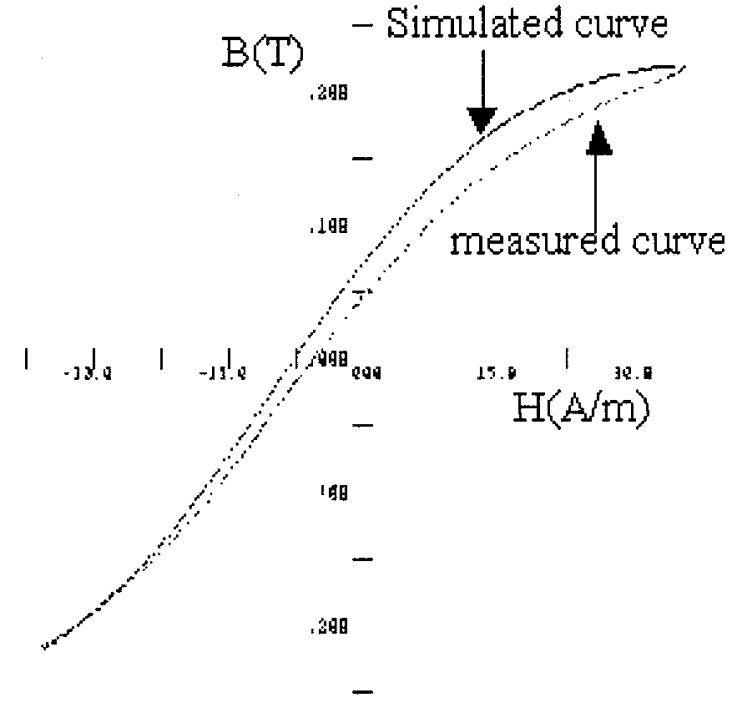

(a)

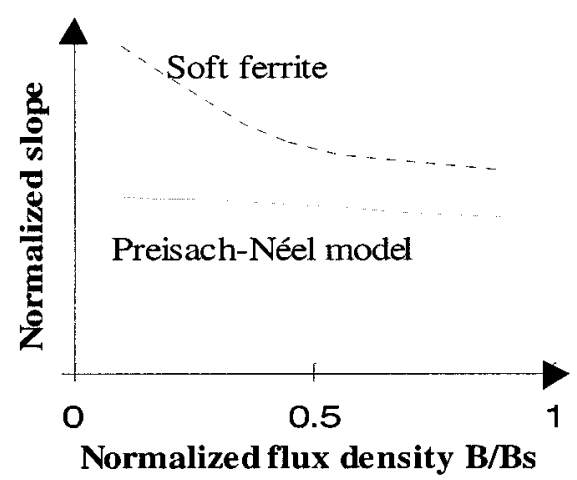

(b)

Fig. 4. (a) Measured and simulated descending hysteresis branch. (b) Normalized slope versus the normalized peak flux density.

termined by the Preisach model are nearly constant (irreversible phenomena), whereas the slopes after the turning points in the case of soft ferrites vary significantly with magnetization. Fig. 4(b) shows the typical value of the normalized slope versus peak flux density, as calculated by the Preisach-Néel model and measured on a soft ferrite. To improve the model for soft ferrite applications, a method was presented in [17]. The approach to correct the Preisach-Néel model is based on the 

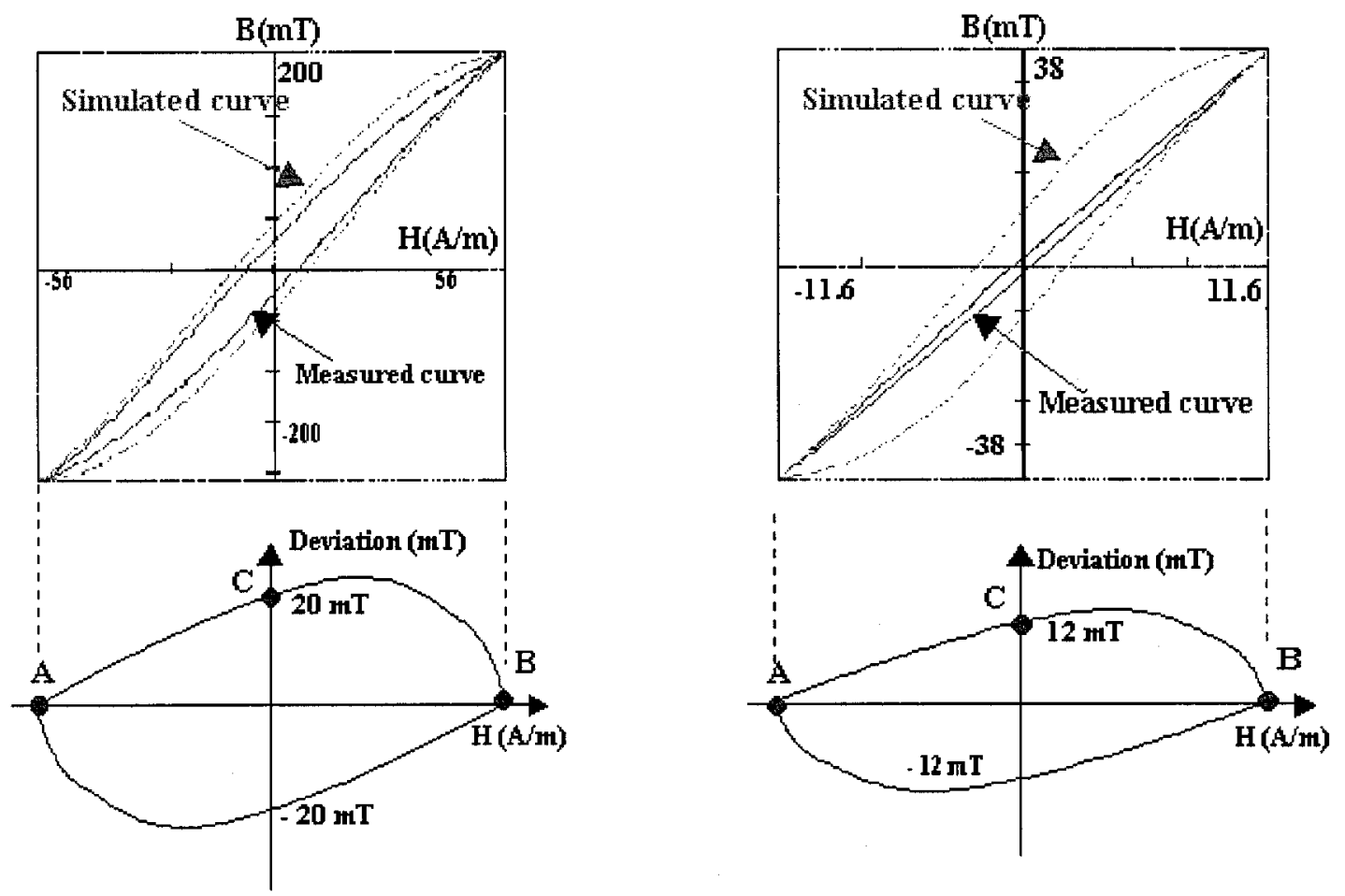

Fig. 5. Comparison between simulated and measured hysteresis loops and the corresponding deviation for two values of the applied field.

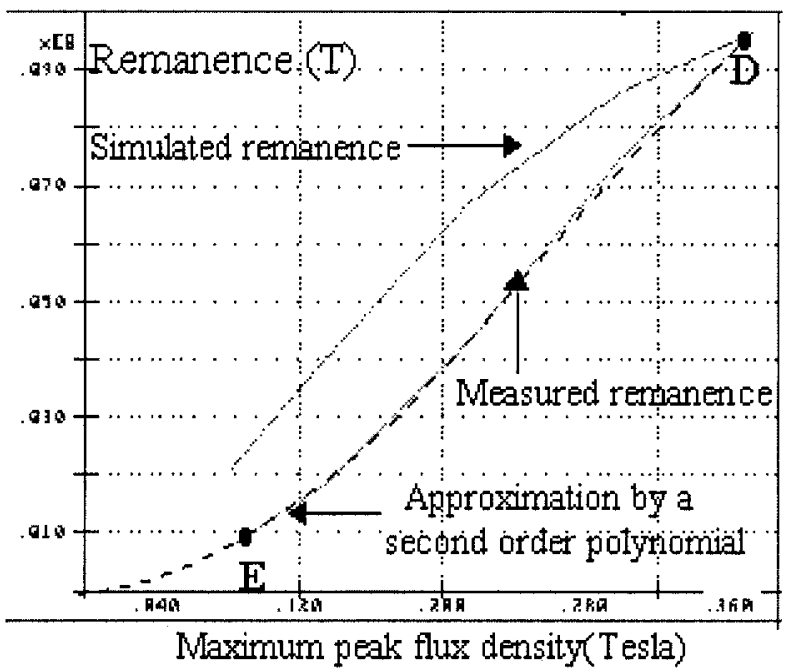

Fig. 6. Comparison between measured and simulated remanence.

analysis of the deviation between simulation and experiment. The deviation is defined as

$$
d(H)=B_{\text {sim }}(H)-B_{\text {meas }}(H)
$$

where $B_{\text {sim }}(H)$ and $B_{\text {meas }}(H)$ are the simulated and the measured flux density, respectively.

Fig. 5 shows that for a soft ferrite material under symmetric excitation, the deviation characteristic has the same form and may be accurately computed for different values of the applied field. These characteristics may be approximated by simple analytical functions (first- and second-order polynomials). Finally,

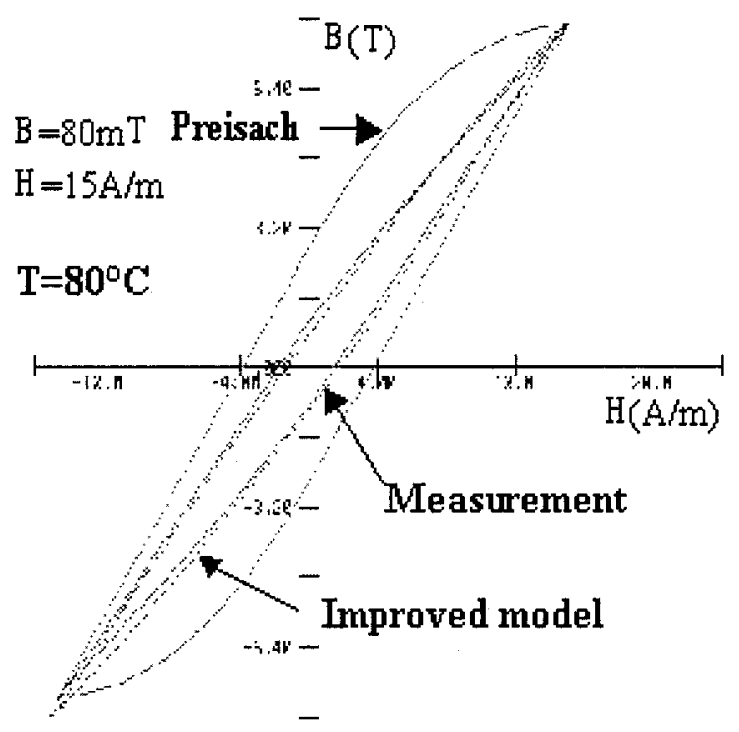

Fig. 7. Validation of the static model at fixed temperature at low flux density level.

the deviation characteristic, for any symmetric loop, may be identified by three points, the turning points $\mathrm{A}$ and $\mathrm{B}$, which are very accurately computed by the Preisach-Néel model and the point $\mathrm{C}$, which is obtained through the deviation between the simulated and the measured remanence. Fig. 6 gives the comparison between the simulated and the measured remanence versus the maximum flux density. The remanence deviation is calculated using the following equation:

$$
d_{\mathrm{Br}}=B_{\mathrm{rsim}}-B_{\mathrm{rmeas}}
$$




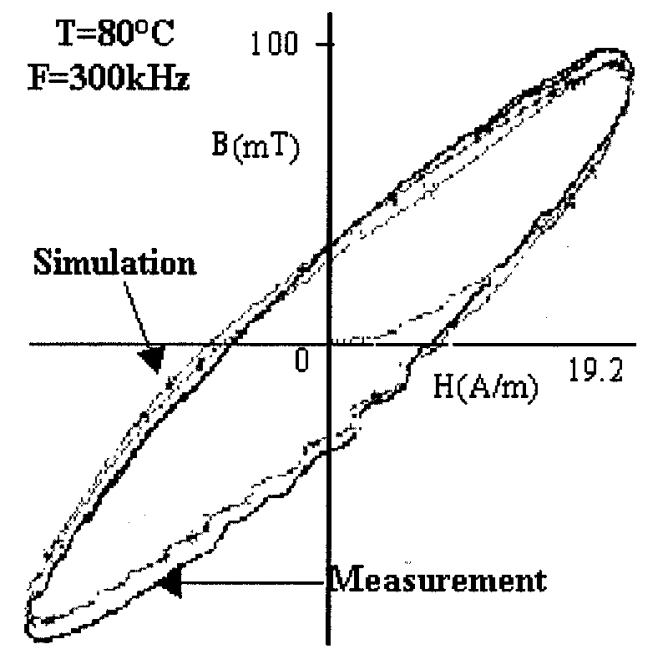

Fig. 8. Validation of the dynamic model at fixed temperature with a triangular excitation (measured losses $=596 \mathrm{~mW} / \mathrm{cm}^{3}$ —computed losses $=$ $590 \mathrm{~mW} / \mathrm{cm}^{3}$.

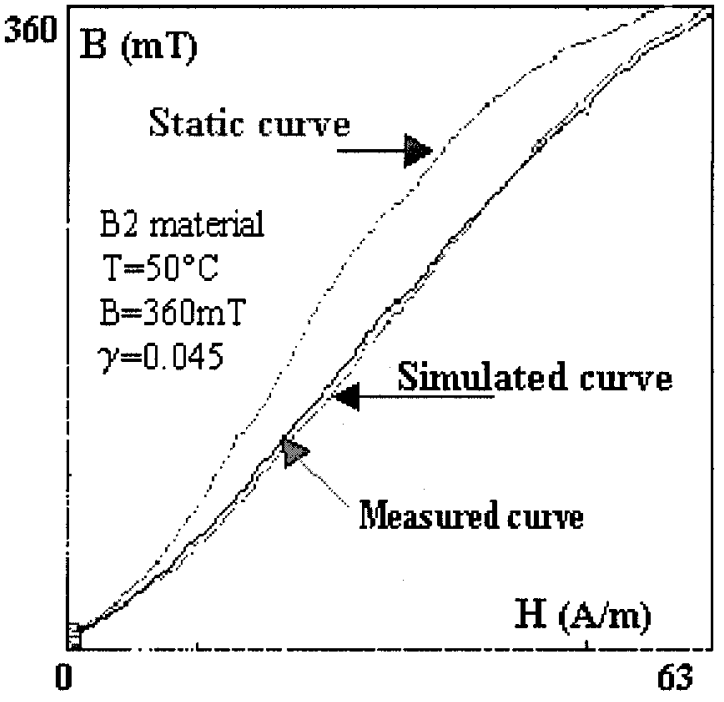

Fig. 9. Identification of the behavioral coefficient at $50^{\circ} \mathrm{C}$.

where $B_{\text {rsim }}$ and $B_{\text {rmeas }}$ represent the simulated and the measured remanence, respectively. The experimental curve may be easily fitted by a second-order polynomial that crosses the origin: two experimental points are then sufficient for its identification. A saturation hysteresis loop (which has been used to identify the Preisach function) and a minor hysteresis loop gives these two points (D and E in Fig. 6). The characteristic of the simulated remanence may be obtained through some simulations and does not need additional data. Therefore, it is easy to obtain an analytical expression of the remanence deviation versus the flux density. As a result, the static model is a combination of both the Preisach-Néel model and the deviation model. In this case, the flux density is given by the following expression:

$$
B(H)=B_{\mathrm{PN}}(H)-B_{d}(H)
$$

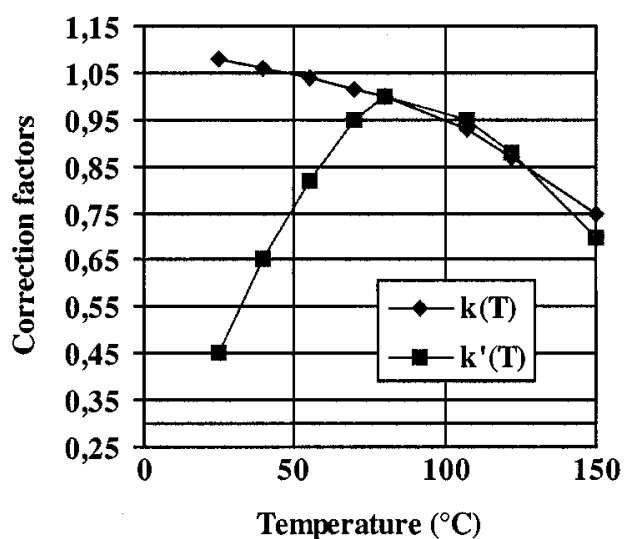

Fig. 10. $k(T)$ and $k^{\prime}(T)$ versus temperature.

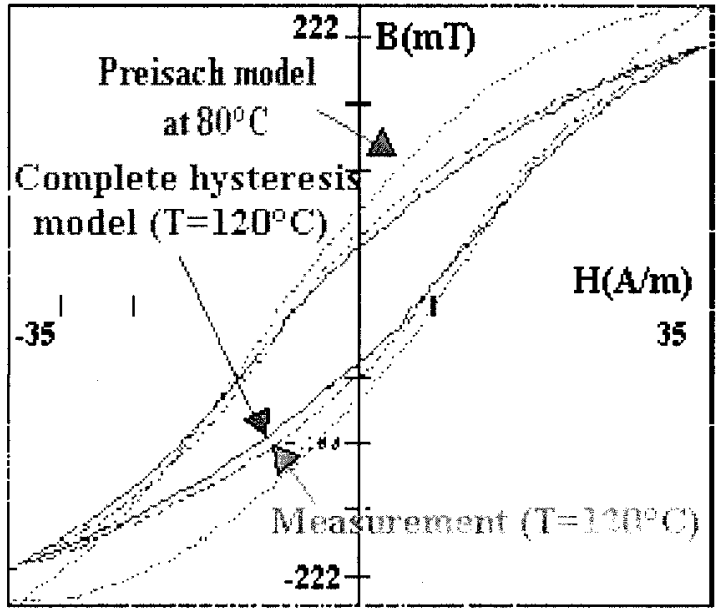

(a)

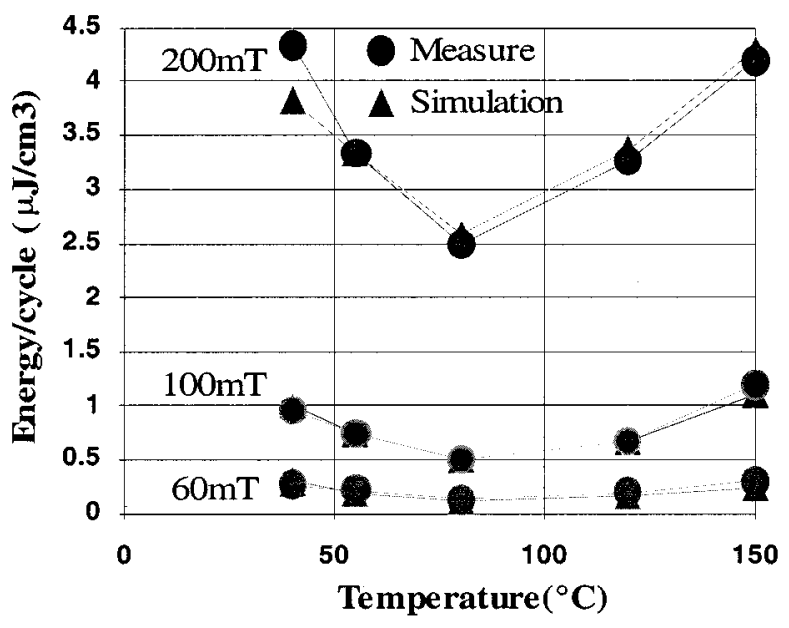

(b)

Fig. 11. Validation of the hysteresis model. (a) Measured and simulated hysteresis loops. (b) Measured and simulated hysteresis losses versus temperature.

where $B_{\mathrm{PN}}(H)$ is the flux density calculated by the Preisach-Néel model and $B_{d}(H)$ is the predicted deviation. 
TABLE I

COMPLETE SimULATION PROGRAM

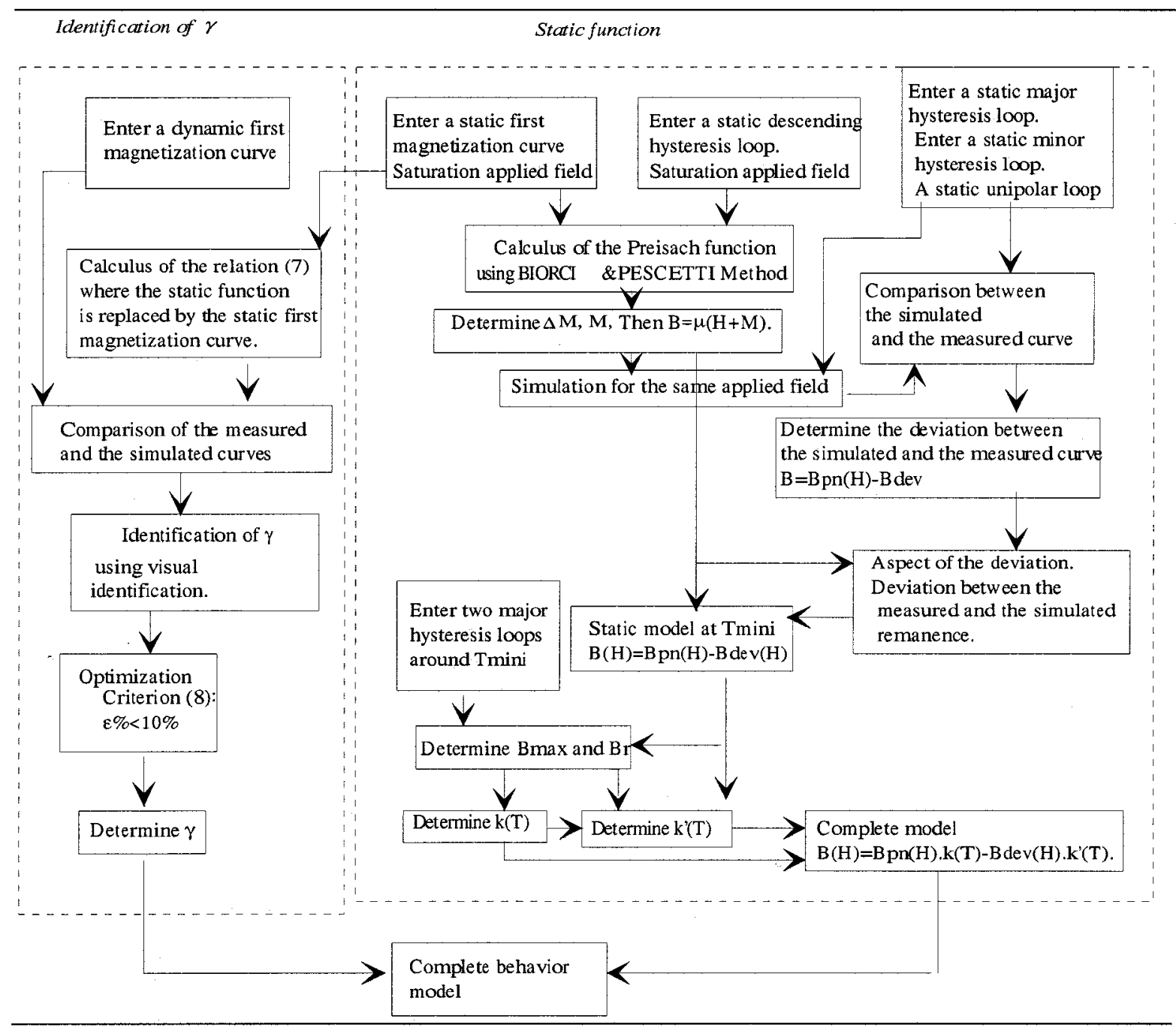

TABLE II

TESTED MATERIALs

\begin{tabular}{l|l|l|l}
\hline Material & $\begin{array}{l}\text { Working frequency } \\
\text { range }(\mathrm{kHz})\end{array}$ & $\begin{array}{l}\text { Temperature of } \\
\text { minimum losses }\left({ }^{\circ} \mathrm{C}\right)\end{array}$ & Curie temperature \\
\hline B3 & $10-50$ & $85-110$ & $250^{\circ} \mathrm{C}$ \\
\hline B4 & $10-70$ & $50-85$ & $200^{\circ} \mathrm{C}$ \\
\hline B2 & $50-250$ & $80-100$ & $250^{\circ} \mathrm{C}$ \\
\hline
\end{tabular}

\section{Application of the Complete Model at a Fixed Temperature}

The behavioral model that takes into account static and dynamic phenomena has been tested in power electronic conditions. It gives accurate values of the iron losses with less than $10 \%$ error and the characteristics $B(H), \varphi(t)$, and $d \varphi / d t$. It requires few parameters and is not time consuming [18].

\section{A Behavioral Model Taking into Account THE TEMPERATURE}

Before presenting the complete model, it is useful to investigate the effect of temperature on both the behavioral coefficient and the static function $B(H)$. The approach consists in introducing the temperature as a variable into the model, previously developed at fixed temperature, without increasing its complexity. The parameters of the model are determined at the temperature value that corresponds to minimum loss. To describe the influence of temperature on magnetic characteristics, a study on the variation of both parameters versus the temperature has been carried out. Both parameters have been tested separately.

\section{A. Influence of Temperature on the Behavioral Model}

The behavioral coefficient has been identified at the temperature of minimum losses as explained in Section II-B, and the same procedure is applied at different temperatures ranging 


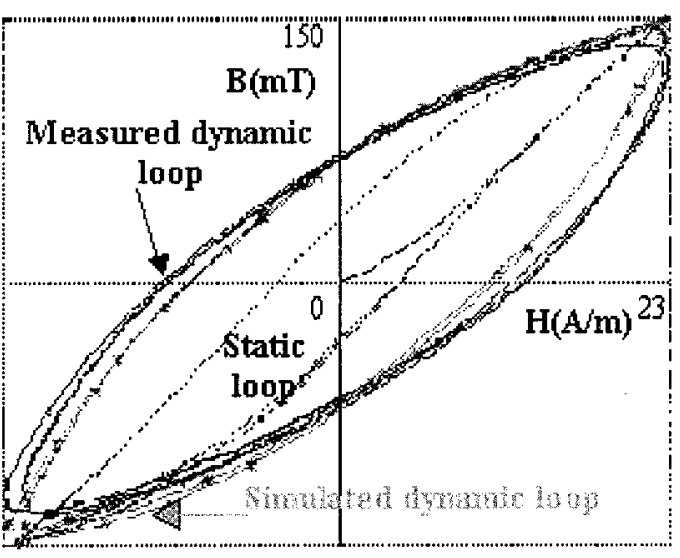

(a)

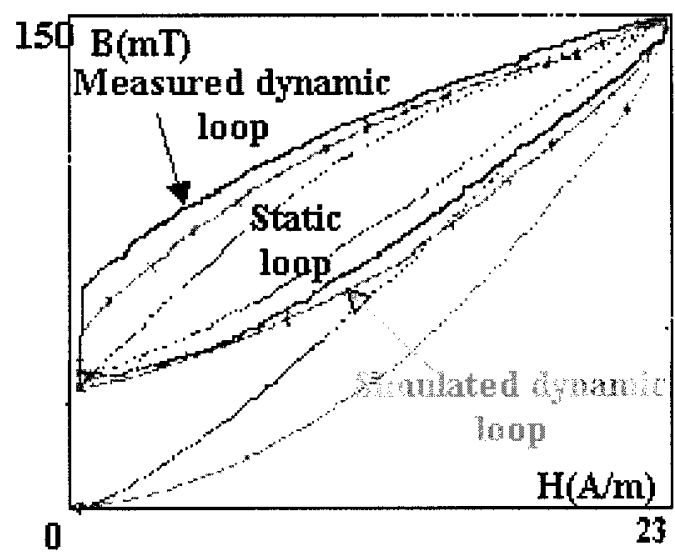

(c)

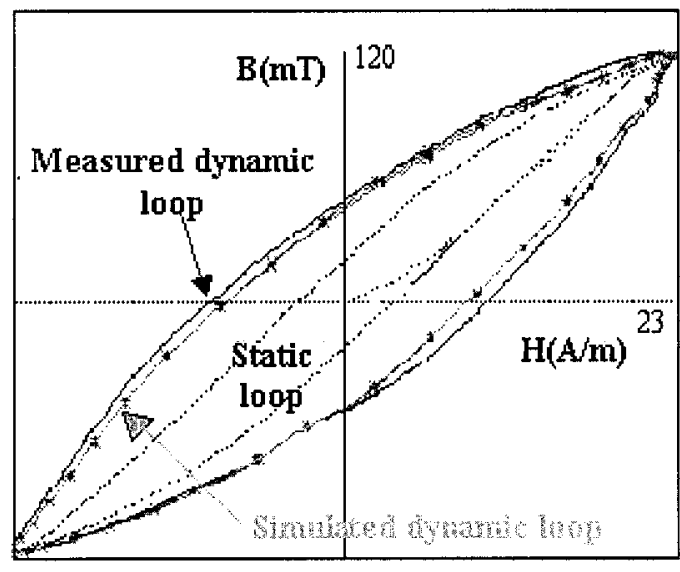

(b)

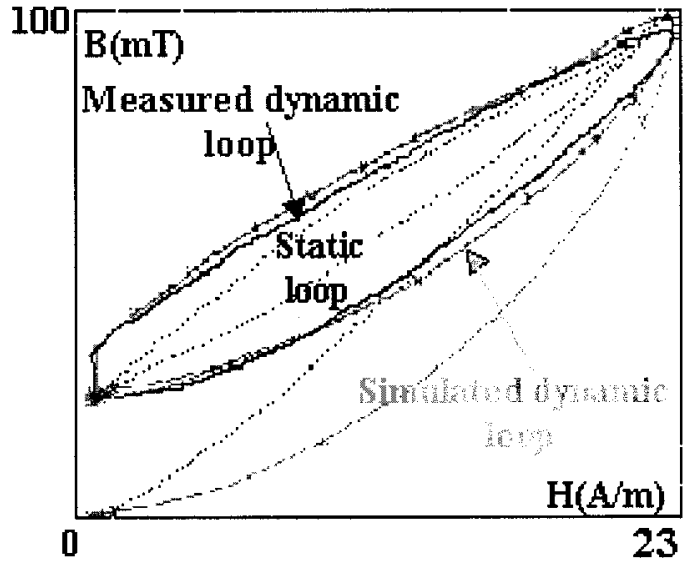

(d)

Fig. 12. Validation of the model for different operating conditions. (a) Symmetric loops at $T=40^{\circ} \mathrm{C}, F=100 \mathrm{kHz}$. (b) Symmetric loops at $T=120{ }^{\circ} \mathrm{C}$, $F=100 \mathrm{kHz}$. (c) Asymmetric loops at $T=40^{\circ} \mathrm{C}, F=100 \mathrm{kHz}$. (d) Asymmetric loops at $T=140{ }^{\circ} \mathrm{C}, F=100 \mathrm{kHz}$.

from 40 to $140^{\circ} \mathrm{C}$. As a result, a good agreement has been found between the measured and the simulated dynamic first curves. As an example, Fig. 7 shows the results obtained at $80{ }^{\circ} \mathrm{C}$ with the same behavioral coefficient ( 0.045 for the B2 material). The behavioral coefficient $\gamma$ appears independent of inputs (waveforms, frequency, and temperature).

\section{B. Influence of Temperature on the Static Function}

The variation of the static function versus temperature is considered at low frequency. As a result, 1) for the same value of the applied field, the increase in temperature decreases all of the peak flux density $(B m)$, the coercive force $(H c)$, the remanence $(B r)$, and the hysteresis losses (Fig. 8); 2) for a fixed value of flux density, the characteristic of hysteresis losses versus the temperature exhibits a minimum at a temperature that corresponds to the temperature of maximum permeability (Fig. 9).

The parameters of the model are identified at the temperature of minimum loss. We have investigated the variation of the flux density estimated by the Preisach-Néel model and the variation of the deviation versus the temperature. Two corrections have been easily implemented in the static model [19].
1) Preisach Model versus Temperature: The first correction concerns the flux density that is calculated by the Preisach-Néel model.

The Preisach-Néel model computes the change of magnetization as follows:

$$
\Delta M=2 . M_{s} \iint \varphi\left(H^{+}, H^{-}\right) d H^{+} d H^{-}
$$

where $M_{s}$ is the saturation magnetization and $\varphi\left(H^{+}, H^{-}\right)$is the Preisach function. The temperature affects both the saturation magnetization and the Preisach function. The aim is to calculate the flux density at any temperature by using a simple alge-braic law. This law is introduced on the saturation magnetization $\Delta M_{s}$; the Preisach function is then taken independent of temperature. The flux density is determined as

$$
B_{T}(H)=B_{T_{\min i}}(H) . k(T)
$$

where $B_{T_{\min i}}(H)$ is the flux density versus the applied field calculated at the temperature of minimum losses and $k(t)$ is the 


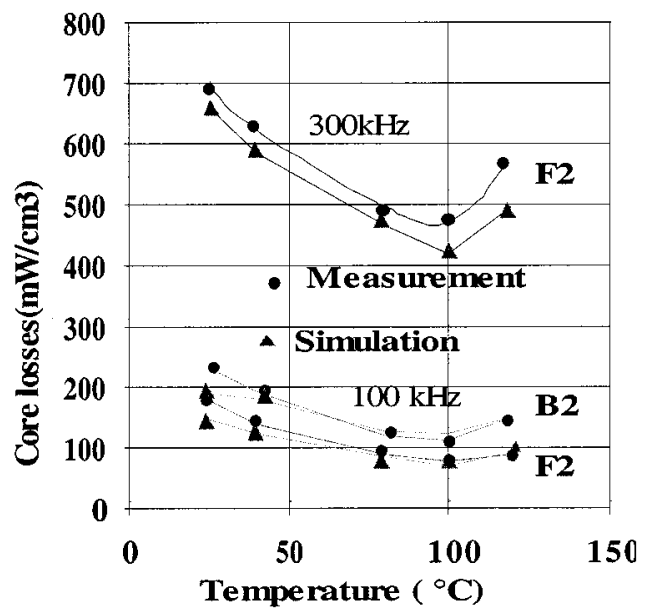

(a)

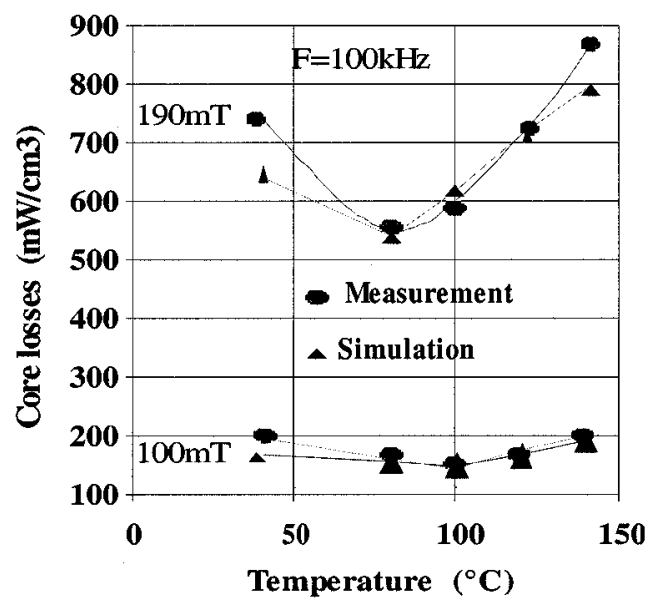

(b)

Fig. 13. Validation of the core losses model. (a) Measured and computed core losses for sine wave excitations. (b) Measured and computed losses for triangular excitations.

correction factor. The latter is obtained through the evolution of the saturation flux density versus the temperature

$$
k(T)=\frac{B_{s m T}}{B_{s m T_{\min i}}} .
$$

This is a function of the saturation flux density measured at the temperature $T, B_{s m T}$, and the saturation flux density measured at the temperature of minimum losses, $B_{S m_{T \min i}}$. This law enables accurate calculations of the maximum flux density, but does not improve the estimation of the remanence and the coercive force [20].

2) Deviation Term versus the Temperature: The analysis of the variation of the deviation model versus the temperature has also been carried out. It may be shown that inside the operating range $\left(40-140^{\circ} \mathrm{C}\right)$, the deviation keeps approximately the same shape. In the operating temperature range, any curve may be obtained from the reference temperature curve by multiplying it by a coefficient $k^{\prime}(T)$ that depends only on the temperature. This law may be identified from data related to any value of $B$. Thus, the remanence point is chosen. This law $k^{\prime}(T)$ is obtained from the curve of remanence versus the temperature and is given by the following equation:

$$
k^{\prime}(T)=\frac{k(T) \cdot B_{r m_{T_{\min i}}}}{B r m_{T}}
$$

where $B_{r m_{T_{\min i}}}$ is the remanence measured at the temperature of minimum losses, $B_{r m_{T}}$ is the remanence measured at a temperature $T$, and $k(T)$ is the coefficient introduced on the Preisach model. The change of both the coefficients $k^{\prime}(T)$ and $k(T)$ versus the temperature are illustrated in Fig. 10. We can see that both coefficients $k(T)$ and $k^{\prime}(T)$ may be well described by a second-order polynomial.

3) Complete Hysteresis Model: The complete hysteresis model is given by

$$
B_{T}(H)=B_{\Gamma N T_{\min i}}(H) \cdot k(T)-k^{\prime}(T) \cdot B_{d_{T \min i}} .
$$

It takes into account the temperature from 40 to $140{ }^{\circ} \mathrm{C}$. It requires three measured hysteresis loops. In order to estimate the behavior of the model, two different points were studied. The main values that have been analyzed [Fig. 11(a)] are the shape of the hysteresis loop, the flux density at a peak applied field, the remanence, and the coercive force. The loop area has also been studied [Fig. 11(b)]. The latter gives the energy dissipated during a cycle. Good agreement has been found.

\section{Application of the Complete Behavioral Model}

The entire composite model comprises the fixed-temperature model [Fig. 2(c)], plus the behavioral laws that correct the Preisach-Néel model or take care of the temperature dependencies. As the model describes parts where the energy is of different nature (electrical, magnetic, thermal), it is suitable to represent the composite model with a bond graph [21] instead of a Kirchhoff network. First, bond graphs offer a more comprehensive graphical representation of the model, but so far without changing anything to the model equations. Second bond graphs present advantages from simulation point of view with respect to the classical Nodal (Modified) Approach implemented in classical circuit simulators. Because bond graphs do not improve the model in any way, and are out of the scope of the present paper, this aspect will not be discussed. Nevertheless, some details are given in the Appendix.

The equations of the bond graph components are implemented in $\mathrm{C}++$ source code. The composite model is simulated with the bond graph simulator PACTE [2], available as the commercial product PowerBond by Dolphin Integration (Grenoble, France). Anyway, the model equations may be adapted for conventional circuit simulators.

The entire parameter evaluation procedures are given in Table I. The model is suitable to predict the iron losses and both the magnetic and electrical characteristics. To demonstrate the overall behavior of the model, several tests were performed on three different soft ferrites. The shapes of the waveforms, the iron losses, and the time evolution of both electrical and magnetic characteristics were analyzed between 40 to $140{ }^{\circ} \mathrm{C}$ and for the classic operating range of soft ferrites.

In order to illustrate the validity of the method, a comparison between measured and calculated values of magnetic and 


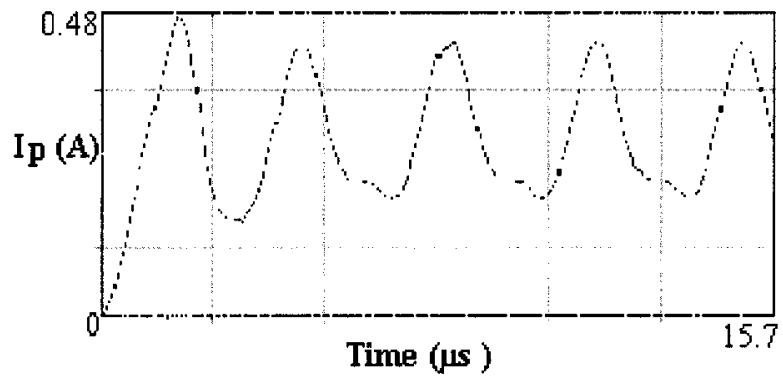

(a)

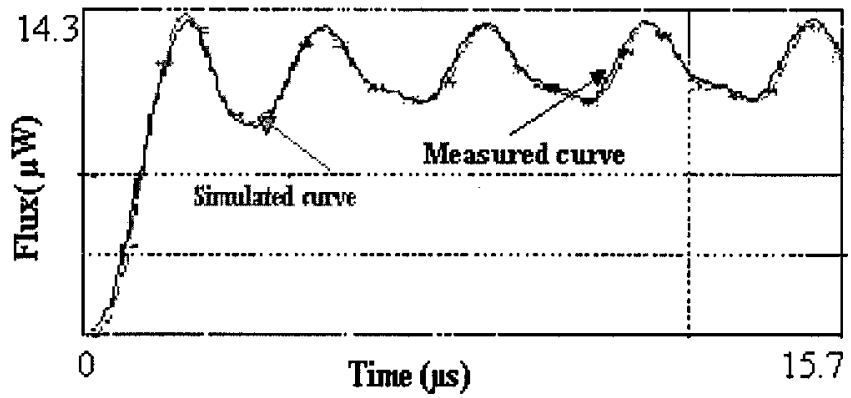

(b)

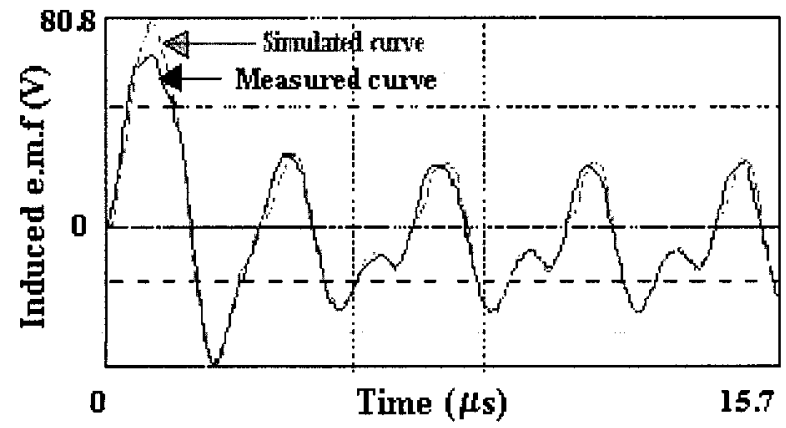

(c)

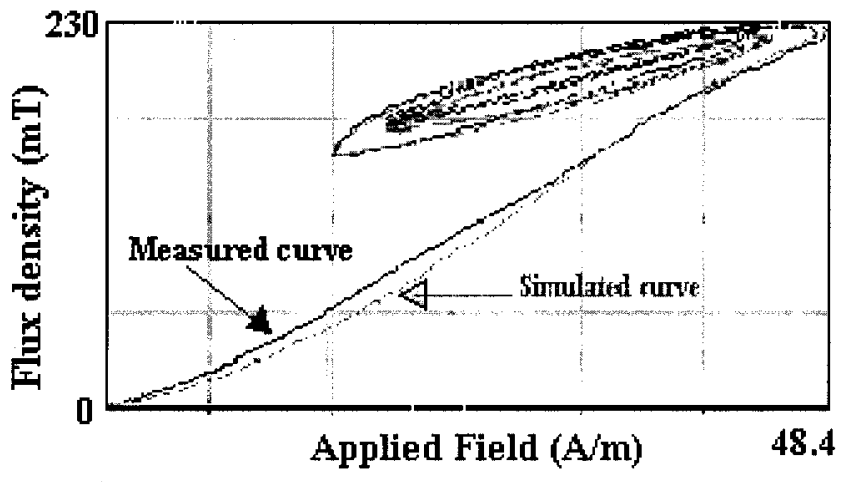

(d)

Fig. 14. Transient working. (a) Excitation waveform. (b) Measured and simulated flux. (c) Measured and simulated induced e.m.f. (d) Measured and simulated loops.

electrical variables is presented. To avoid using too many figures, only the results related to one material are shown. The considered material is FERRINOX B2 $(70 \mathrm{kHz}-250 \mathrm{kHz})$ by LCC
Thomson-CSF, which is a "low-loss power material." It offers low losses above $80^{\circ} \mathrm{C}$ [22]. It may be noted that four materials were tested (Table II).

The first comparisons [Figs. 12(a) and (b)] concern steady state under symmetrical triangular excitation and two temperatures 40 and $120{ }^{\circ} \mathrm{C}$ with a frequency equal to $100 \mathrm{kHz}$ (push-pull application).

The second case concerns steady state under asymmetrical triangular excitation and two temperatures 40 and $140{ }^{\circ} \mathrm{C}$ with a frequency equal to $100 \mathrm{kHz}$ (flyback and forward application) [Fig. 12(c) and (d)].

In the symmetric or asymmetric loops, we notice the good behavior of the model to describe the major and minor loops. The model accurately describes the core losses (less than 20\%).

The third case concerns the comparison between the losses measured by the manufacturer and the simulated losses under sine wave excitation source and a peak flux density of $100 \mathrm{mT}$ and two frequencies: $100 \mathrm{kHz}$ and $300 \mathrm{kHz}$ [Fig. 13(a)]. The considered materials are F2 $(100-500 \mathrm{kHz})$ and B2 $(70-250$ $\mathrm{kHz}$ ).

The fourth case shows the comparison between the measured and the simulated core losses, for two values of flux density (100 and $190 \mathrm{mT}$ ). The excitation source is triangular, and the frequency is $100 \mathrm{kHz}$ [Fig. 13(b)].

The last one is related to transient operation under unipolar excitation with a high DC component, with a previously demagnetized material (Fig. 14). The induced voltage is calculated accurately, even though it has been obtained by computing the flux derivative with respect to time.

All of these simulations are performed with the same behavioral coefficient value $\gamma$ characterizing here the B2 Thomson material. The temperature was introduced in the static function. In the symmetric or asymmetric loops, we notice the good behavior of the model to describe the major and minor loops. The model also describes accurately the core losses (error less than $20 \%)$.

1) Measurement Techniques: Two windings are placed on the core under test. The first winding $(N p)$ serves as the excitation winding, whereas the second winding $(\mathrm{Ns})$ is used to sense the induced voltage caused only by the rate of change of flux in the secondary winding. To validate the proposed model and considering that the practical cores used in power applications have a cross section that is not small or uniform, the core under test is characterized by its effective dimensions [4] [effective magnetic length $l_{e}=\left(2 \pi \log _{e}\left(r_{2} / r_{1}\right) /\left(1 / r_{1}\right)-\left(1 / r_{2}\right)\right)$ and effective cross-sectional area $S_{e}=\left(h \log _{e}^{2}\left(r_{2} / r_{1}\right) /\left(1 / r_{1}\right)-\right.$ $\left.\left.\left(1 / r_{2}\right)\right)\right]$. These effective dimensions would define a hypothetical toroid having the same properties as the uniform core considered in the model. To obtain the core material losses and the electrical and magnetic characteristics, our approach consists of measuring the applied field in the primary winding: $H=\left(N_{p} \cdot I_{1} / 1_{e}\right)$ and the induced voltage $v_{s}$. The induced voltage $v_{s}$ at the secondary winding is integrated to obtain the flux density $B=\left(1 / N_{s} . S_{e}\right) \int_{0}^{t} v_{s}(t) d t$. The area of the $B$ versus $H$ loop is equal to the energy lost per cycle per unit volume $\left(V_{e}\right), W=\left(N_{1} / N_{2} V_{e}\right) \int_{0}^{t} I_{1}(t) \cdot v_{s}(t) d t$.

The experimental setup uses a high linear power amplifier to obtain a controlled current excitation, driven by a function gen- 


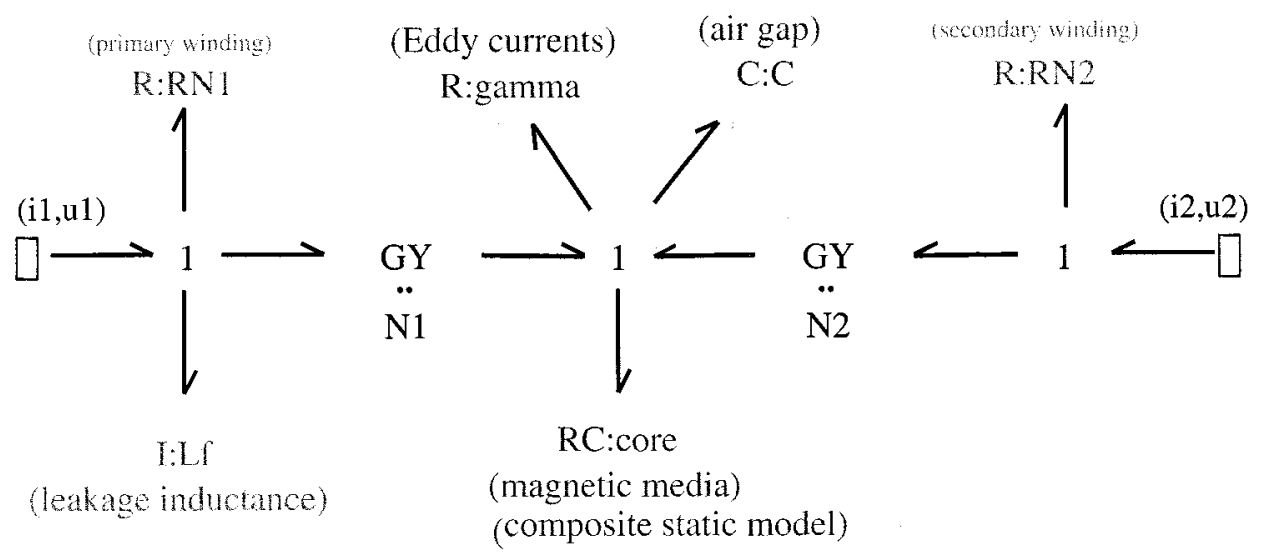

Fig. 15. Generalized bond graph of a two-winding ferrite magnetic component.

erator to provide variable excitation voltage, frequency, and amplitude of the desired waveforms for testing the magnetic cores. The acquisition of the primary current using a coaxial shunt resistor and the secondary voltage are plotted on an oscilloscope type: TEKTRONIX TDS 540.

The core heating is obtained by a regulated air flow. The measurement is performed after a period of time sufficient for the core to reach a thermal equilibrium. The core temperature is assumed to be homogeneous during the measurement. To validate the measurements, a comparison with the manufacturer's core losses data in the case of sine waves was performed and a good agreement was obtained. The results are similar for the tested materials.

\section{CONCLUSION}

Together with the magnetic field (frequency, waveform, and flux density), the temperature is an important factor of changes in both magnetic and electrical characteristics of soft ferrites used as magnetic material in power conversion. A model for describing the behavior of soft ferrites, including the temperature, has been presented. The model may be used in different conditions encountered in power electronics. The temperature has been introduced in the behavioral model by using empirical laws that are easily identified by simple quasi-static measurements. The model requires only a few parameters, and it proves to be easy to use. The accuracy of the model is $20 \%$ in a wide range of frequencies and temperatures.

\section{APPENDIX}

A two-winding ferrite magnetic component may be represented by the bond graph in Fig. 15. Bond graphs [21] picture the flow, the storage, and dissipation of energy through a system. A flow of energy - whatever is the physical domain —is pictured by a bond terminated by an half-arrow. A bond is characterized by a flow variable and an effort variable that depend on the physical domain, but their product is always a power. For example, the electrical domain is characterized by the current as the flow variable $(\boldsymbol{i})$ and the voltage drop as the effort variable $(\boldsymbol{u})$. The magnetic domain is characterized by the time-derivative of the magnetic flux as the flow variable $(\lambda)$ and the difference in magnetic potential as the effort variable $(\boldsymbol{F})$.

The element noted "GY" is a gyrator that fixes the energy transformation between electrical and magnetic domains. The element noted " 1 " is a one-junction that denotes the equality of the flow variable in all of the connected components.

The right part of the bond graph in Fig. 15 is not relevant in the present paper, as the model is described in the case of a component with only one winding. The static part of the entire composite model is represented by an $R C$-element, which means that the element has a dissipator behavior mixed with a storage behavior. The dynamical part of the composite model is represented by the $R$-element related to $\gamma$, and a $C$-element related to the air-gap.

From a modeling point of view, the equations of the different elements, as discussed in this paper, are not changed by the bond graph approach. The main benefit of the bond graph approach is the notion of causality. Basically, a model of an element has several inputs (flow and effort variables) and calculates the values of outputs (dual effort and flow variables). It means that the element inputs are imposed by the outside world, and the element reacts by controlling the outputs. These cause-to-effect relations are called the causality. The causality helps in understanding the role of each element of a system. A Kirchhoff network does not clearly show the model causality.

From a simulation point of view, the causality analysis, i.e., the analysis of the causality throughout a bond graph, helps writing the set of equations that must be computed in the most efficient way, and not an implicit matter obligatorily, as does the Nodal Approach.

\section{ACKNOWLEDGMENT}

The authors wish to thank P. Mas (SCHNEIDER ELECTRIC) and W. Morweiser (LCC THOMSON-CSF) for their many valuable suggestions.

\section{REFERENCES}

[1] Saber User Guide. Beaverton, OR: Analogy Inc..

[2] B. Allard, H. Morel, S. Ghedira, and A. Ammous, "A bond graph simulator including semiconductor device models," in Proc. CESA'96, IMACS Multiconf., vol. 1, p. 500. 
[3] M. Bogs, M. Esguerra, and W. Holubarsh, "New ferrite material for high frequency power transformers," in Proc. Power Conversion, Munich, Germany, 1993, pp. 361-370.

[4] E. C. Snelling, Soft Ferrites: Properties and Applications, 2nd ed. London: Butterworth, 1988 .

[5] L. Zegadi, J. J. Rousseau, and P. Tenant, "Prise en compte de la temperature dans un modele d'hysteresis pour ferrites doux MnZn," J. Phys. III, vol. 7, pp. 369-385, 1997.

[6] B. Carsten, "High frequency conductor losses in switchmode magnetics," in Proc. High Frequency Power Conf., 1986, pp. 155-176.

[7] J. A. Ferreira, "Electromagnetic modeling of power electronic converter under conditions of appreciable skin and proximity effects," Dr. Eng. thesis, Rand Afrikaans Univ., South Africa, 1987.

[8] A. W. Lotfi, P. M. Gradzky, and F. C. Lee, "Proximity effects in coils for high frequency power applications," IEEE Trans. Magn., vol. 28, pp. 2169-2171, Sept. 1992

[9] F. Preisach, "Uber die magnetische nackwirkung," Zeitschrift für Physik, vol. 94, pp. 277-302, 1935

[10] D. C. Jiles and D. L. Atherton, "Theory of ferromagnetic hysteresis," J. Appl. Phys., vol. 25, pp. 2115-2119, 1984

[11] J. J. Rousseau, B. Lefevbre, and J. P. Masson, "Behavioral model of iron losses," in Int. Math. Comput. Simul., 1990, pp. 431-436.

[12] F. Marthouret, J. P. Masson, and H. Fraisse, "Modeling of a nonlinear conductive magnetic circuit. Part 1; definition and experimental validation of an equivalent problem," IEEE Trans. Magn., vol. 31, pp. 4065-4067, Nov. 1995.

[13] G. Biorci and D. Pescetti, "Analytical theory of the behavior of ferromagnetic materials," Nuovo Cimento, vol. 7, pp. 1551-1557, 1958.

[14] E. Della Torre, J. Oti, and G. Kadar, "Preisach modeling and reversible magnetization," IEEE Trans. Magn., vol. 26, pp. 3052-3058, Nov. 1990.

[15] D. L. Atherton, B. Szpunar, and J. A. Szpunar, "A new approach to Preisach diagrams," IEEE Trans. Magn., vol. M-23, pp. 1856-1865, May 1987.

[16] O. Benda, "To the question of the reversible processes in the Preisach Model," Electrotechnic, vol. Cas 42, pp. 186-191, 1991.

[17] J. J. Rousseau, P. Tenant, and L. Zegadi, "Improvement of Preisach Model. Application to soft magnetic materials," J. Phys. III, pp. 1717-1727, Aug. 1997.

[18] P. Tenant and J. J. Rousseau, "Dynamic model for soft ferrites," in Proc. IEEE Power Electron. Specialists Conf., vol. 2, Atlanta, GA, 1995, pp. $1070-1076$.
[19] J. J. Rousseau, L. Zegadi, and P. Tenant, "A complete hysteresis model for soft ferrites," in Proc. IEEE Power Electron. Specialists Conf., vol. 1, Baveno, Italy, 1996, pp. 322-328.

[20] P. Tenant, J. J. Rousseau, and L. Zegadi, "Hysteresis modeling taking into account the temperature," in Proc. 6th Eur. Conf. Power Electron. Applicat., vol. 1, Seville, Spain, 1994, pp. 1.001-1.006.

[21] D. Karnopp, R. Rosenberg, and D. Margolis, System Dynamics: A Unified Approach. New York: Addison-Wesley, 1991

[22] LCC THOMSON-CSF, “Soft ferrites,”, Beaune, France, 1994.

Jean-Jacques Rousseau was born in 1953. He received the E.E. degree in 1979 and the Ph.D. degree in 1983, both from the University of Clermont-Ferrand, Clermond-Ferrand, France.

He is currently a Full Professor at University of Saint-Etienne, Saint-Etienne, France. He has been with the Electrical Engineering Center of Lyon (CEGELY), Villeurbanne, France, since 1987. His current research interests include power electronics and magnetic component modeling.

Bruno Allard (M'92) received the engineering, M.S., and Ph.D. degrees from the Institut National Des Sciences Appliquées de Lyon (INSA Lyon), Lyon, France, respectively in 1988, 1989, and 1992.

$\mathrm{He}$ is currently with the Electrical Engineering Center of Lyon (CEGELY) and Professor at the Electrical Engineering Department of INSA Lyon. His research interests include advanced electrothermal modeling of power devices, power converter simulation using bond graphs, $\mathrm{CAD}$ of integrated power converters.

Pierre Tenant was born in 1966. He received the Ph.D. degrees from the Institut National Des Sciences Appliquées de Lyon, Lyon, France, in 1995.

He was with the Electrical Engineering Center of Lyon (CEGELY) from 1991 to 1995 . His research interests include the modeling of magnetic phenomena. Since 1996, he has been with ISOLEC, Villeurbanne, France, where he works on transformer design. 